\title{
IDEALS OF SEMIRINGS USING MULTISET THEORY
}

\author{
DEBABRATA MANDAL \\ Department of Mathematics, Raja Peary Mohan College, Uttarpara, Hooghly-712298, India \\ *Email Address: dmandaljumath@gmail.com \\ Received May 04, 2019

\begin{abstract}
Aвstract. The concept of multi ideal, multi bi-ideal, multi quasi-ideal of a semiring are introduced and some of their related properties are investigated. Some characterizations of regular and intra-regular semiring are also obtained. 2010 Mathematics Subject Classification. 16Y99.
\end{abstract}

Key words and phrases. Bi-ideal; Ideal; Multiset; Quasi-ideal; Regular; Semiring.

\section{INTRODUCTION}

There are many concepts of universal algebras generalizing an associative ring $(R,+,$.$) . Some of them - in$ particular, semiring have been found very useful for solving problems in different areas of applied mathematics and information sciences, since the structure of a semiring provides an algebraic framework for modelling and studying the key factors in these applied areas. Ideals of semiring play a central role in the structure theory and useful for many purposes.

Multisets is an important generalization of classical set theory which has emerged by violating a basic property of classical sets that an element can belong to a set only once. This set theory has various applications in mathematics and computer science, overview of which can be obtained in [9]. Many authors, like $[1-3,5-7,10]$ etc. have enriched the theory of multisets. Nazmul et al [8] applied this to the theory of groups.

As a generalization of this, in this paper we have introduced the concept of multi ideal in the theory of semirings and investigated some of its related properties.

\section{Preliminaries}

We recall the following preliminaries for subsequent use.

Definition 2.1. [4] A hemiring [respectively semiring] is a nonempty set $S$ on which operations addition and multiplication have been defined such that the following conditions are satisfied:

(i) $(S,+)$ is a commutative monoid with identity 0.

(ii) $(S,$.$\left.) is a semigroup [respectively monoid with identity 1_{S}\right]$.

(iii) Multiplication distributes over addition from either side.

(iv) $0 . s=0=s .0$ for all $s \in S$.

DOI: $10.28924 /$ APJM/6-20

(92019 Asia Pacific Journal of Mathematics 
(v) $1_{S} \neq 0$

Throughout this paper, unless otherwise mentioned $S$ denotes a semiring. A subset $A$ of a semiring $S$ is called a left(resp. right) ideal of $S$ if $A$ is closed under addition and $S A \subseteq A$ (resp. $A S \subseteq A$ ). A subset $A$ of a semiring $S$ is called an ideal if it is both left and right ideal of $S$.

A subset $A$ of a semiring $S$ is called a bi-ideal if $A$ is closed under addition and $A S A \subseteq A$.

A subset A of a semiring $S$ is called a quasi-ideal of $S$ if $A$ is closed under addition and $S A \cap A S \subseteq A$.

Definition 2.2. [3] A multiset $M$ drawn from the set $X$ is represented by a Count function $C_{M}$ defined as $C_{M}: X \rightarrow N$, where $N$ represents the set of non-negative integers.

Here $C_{M}(x)$ is the number of occurance of the element $x$ in the multiset $M$.

\section{Multi Ideal of Semiring}

Definition 3.1. Let $S$ be a semiring and $x, y \in S$. A multiset $M$ over $S$ called a multi left ideal [resp. multi right ideal] of $S$ if

(i) $C_{M}(x+y) \geq \min \left[C_{M}(x), C_{M}(y)\right]$ and

(ii) $C_{M}(x y) \geq C_{M}(y)\left[\right.$ resp. $\left.C_{M}(x y) \geq C_{M}(x)\right]$

A multi ideal of a semiring $S$ is a non empty multi subset of $S$ which is a multi left ideal as well as a multi right ideal of $S$.

Example 3.2. Let $S$ be the additive commutative semigroup of all integers. Then $S$ is a semiring if ab denotes the usual multiplication of integers $a$ and $b$ where $a, b \in S$. Let $C_{M}$ be a multi subset of $S$, defined as follows

$$
C_{M}(x)=\left\{\begin{array}{l}
7 \text { if } x \text { is even } \\
5 \text { if } x \text { is odd }
\end{array}\right.
$$

The multi subset $C_{M}$ of $S$ is a multi ideal $S$.

Definition 3.3. Let $P$ and $Q$ be two multisets over a semiring $S$. Define multi-composition of $P$ and $Q$ by $C_{P} o C_{Q}(x)=\sup \left[\min _{i}\left\{\min \left\{C_{P}\left(a_{i}\right), C_{Q}\left(b_{i}\right)\right\}\right\}\right]$

$$
x=\sum_{i=1}^{n} a_{i} b_{i}
$$

$=0$, if $x$ cannot be expressed as above

where $x, a_{i}, b_{i} \in S$.

Definition 3.4. Let $P$ and $Q$ be two multisets over a semiring $S$. Define intersection of $P$ and $Q$ by

$$
C_{P} \cap C_{Q}(x)=\min \left\{C_{P}(x), C_{Q}(x)\right\}
$$

Lemma 3.5. Let $P$ and $Q$ be two multi ideal over a semiring $S$. Then $C_{P} o C_{Q} \subseteq C_{P} \cap C_{Q} \subseteq C_{P}, C_{Q}$ 
Proof. $P$ and $Q$ be two multisets over a semiring $S$ with $x \in S$. Then

$$
\begin{aligned}
\left(C_{P} o C_{Q}\right)(x)= & \sup \left\{\min _{i}\left\{\min \left\{C_{P}\left(a_{i}\right), C_{Q}\left(b_{i}\right)\right\}\right\}\right\} \\
& x=\sum_{i=1}^{n} a_{i} b_{i} \\
\leq & \sup \left\{\min _{i}\left\{\min \left\{C_{P}\left(a_{i}\right)\right\}\right\}\right\} \\
\leq & C_{P}\left(\sum_{i=1}^{n} a_{i} b_{i}\right)=C_{P}(x) \ldots \ldots \ldots \ldots \ldots(1)
\end{aligned}
$$

Since this is true for every representation of $x, C_{P} o C_{Q} \subseteq C_{P}$.

Similarly we can prove that

$$
C_{P} o C_{Q} \subseteq C_{Q} \text { for all } x \in S
$$

Combining (1)and (2)we get

$$
\begin{aligned}
\left(C_{P} o C_{Q}\right)(x) & \leq \min \left\{C_{P}(x), C_{Q}(x)\right\} \text { for all } x \in S \\
& =\left(C_{P} \cap C_{Q}\right)(x) \ldots \ldots \ldots(3)
\end{aligned}
$$

Therefore, combining (1), (2) and (3) and we get that

$$
C_{P} o C_{Q} \subseteq C_{P} \cap C_{Q} \subseteq C_{P}, C_{Q}
$$

Hence the lemma.

Proposition 3.6. Intersection of a nonempty collection of multi left ideals is a multi left ideal over $S$.

Proof. Let $\left\{M_{i}: i \in I\right\}$ be a non-empty family of multi left ideals of $S$ and $x, y \in S$. Then

$$
\begin{aligned}
\left(\cap_{i \in I}^{\cap} C_{M_{i}}\right)(x+y) & =\inf _{i \in I}\left[C_{M_{i}}(x+y)\right] \\
& \geq \inf _{i \in I}\left[\min \left[C_{M_{i}}(x), C_{M_{i}}(y)\right]\right] \\
& =\min \left[\inf _{i \in I} C_{M_{i}}(x), \inf _{i \in I} C_{M_{i}}(y)\right] \\
& =\min \left[\left(\cap_{i \in I} C_{M_{i}}\right)(x),\left(\cap_{i \in I} C_{M_{i}}\right)(y)\right] .
\end{aligned}
$$

Again

$$
\begin{aligned}
\left(\cap_{i \in I} C_{M_{i}}\right)(x y) & =\inf _{i \in I}\left[C_{M_{i}}(x y)\right] \\
& \geq \inf _{i \in I}\left[C_{M_{i}}(y)\right] \\
& =\left(\bigcap_{i \in I} C_{M_{i}}\right)(y)
\end{aligned}
$$

Hence $\bigcap_{i \in I} M_{i}$ is a multi left ideal of $S$.

Definition 3.7. Let $R, S$ be semirings and $f: R \rightarrow S$ be a function and $a, b \in R$. Then $f$ is said to be a homomorphism if

(i) $f(a+b)=f(a)+f(b)$

(ii) $f(a b)=f(a) f(b)$

(iii) $f\left(0_{R}\right)=0_{S}$,

where $0_{R}$ and $0_{S}$ are the zeroes of $R$ and $S$ respectively. 
Definition 3.8. Let $X$ and $Y$ be two non-empty sets and $f: X \rightarrow Y$ be a mapping. Then

(i) the image of multiset $P$ over $X$ under the mapping $f$ denoted by $f(P)$ where

$$
C_{f(P)}(y)=\left\{\begin{array}{l}
\sup _{x \in f^{-1}(y)} C_{P}(x) \\
0 \text { otherwise }
\end{array}\right.
$$

(ii) the inverse image of a multiset $Q$ over $S$ under the mapping $f$ is denoted by $f^{-1}(Q)$, where $C_{f^{-1}(Q)}(x)=C_{Q}(f(x))$.

Proposition 3.9. Let $f: R \rightarrow S$ be a morphism of semirings.

(i) If $Q$ is a multi left ideal of $S$, then $f^{-1}(Q)$ is a multi left ideal of $R$.

(ii) If $f$ is surjective morphism and $P$ is a multi left ideal of $R$, then $f(P)$ is a multi left ideal of $S$.

Proof. Let $f: R \rightarrow S$ be a morphism of semirings.

(i) Let $Q$ be a multi left ideal of $S$. Now, for any $r, s \in R$

$$
\begin{aligned}
C_{f^{-1}(Q)}(r+s) & =C_{Q}(f(r+s))=C_{Q}(f(r)+f(s)) \\
& \geq \min \left\{C_{Q}(f(r)), C_{Q}(f(s))\right\} \\
& =\min \left\{\left(C_{f^{-1}(Q)}\right)(r),\left(C_{f^{-1}(Q)}\right)(s)\right\} .
\end{aligned}
$$

Again

$$
\begin{aligned}
\left(C_{f^{-1}(Q)}\right)(r s) & =C_{Q}(f(r s))=C_{Q}(f(r) f(s)) \\
& \geq C_{Q}(f(s))=\left(C_{f^{-1}(Q)}\right)(s) .
\end{aligned}
$$

Thus $f^{-1}(Q)$ is a multi left ideal of $R$.

(ii) Let $P$ be a multi left ideal of $R$. Now, for any $x^{\prime}, y^{\prime} \in S$

$$
\begin{aligned}
\left(C_{f(P)}\right)\left(x^{\prime}+y^{\prime}\right) & =\sup _{z \in f^{-1}\left(x^{\prime}+y^{\prime}\right)}(z) \\
& \left.\geq \sup _{x \in f^{-1}\left(x^{\prime}\right), y \in f^{-1}\left(y^{\prime}\right)}(x)\right] \\
& \geq \sup \left[\min \left[C_{P}(x), C_{P}(y)\right]\right] \\
& =\min \left[\sup C_{P}(x), \sup C_{P}(y)\right] \\
& =\min \left[\left(C_{f(P)}\right)\left(x^{-1}\left(x^{\prime}\right),\left(C_{f(P)}\right)\left(y^{\prime}\right)\right]\right.
\end{aligned}
$$

Again

$$
\begin{aligned}
C_{f(P)}\left(x^{\prime} y^{\prime}\right) & =\sup _{z \in f^{-1}\left(x^{\prime} y^{\prime}\right)} C_{P}(z) \sup _{x \in f^{-1}\left(x^{\prime}\right), y \in f^{-1}\left(y^{\prime}\right)} C_{P}(x y) \\
& \geq \sup _{y \in f^{-1}\left(y^{\prime}\right)} C_{P}(y)=\left(C_{f(P)}\right)\left(y^{\prime}\right) .
\end{aligned}
$$

Thus $f(P)$ is a multi left ideal of $S$.

Let $\left\{S_{i}\right\}_{i \in I}$ be a family of semirings. Now if we define addition (+) and multiplication (.) on the cartesian product $\Pi_{i \in I} S_{i}$ as follows :

$\left(x_{i}\right)_{i \in I}+\left(y_{i}\right)_{i \in I}=\left(x_{i}+y_{i}\right)_{i \in I}$ and $\left(x_{i}\right)_{i \in I}\left(y_{i}\right)_{i \in I}=\left(x_{i} y_{i}\right)_{i \in I}$ for all $\left(x_{i}\right)_{i \in I},\left(y_{i}\right)_{i \in I} \in \Pi_{i \in I} S_{i}$ then $\Pi_{i \in I} S_{i}$ becomes a semiring. 
Definition 3.10. Let $P$ and $Q$ be multi subsets of $X$. The cartesian product of $P$ and $Q$ is defined by $\left(C_{P} \times C_{Q}\right)(x, y)=$ $\min \left\{C_{P}(x), C_{Q}(y)\right\}$ for all $x, y \in X$.

Theorem 3.11. Let $P$ and $Q$ be multi left ideals over a semiring $S$. Then cartesian product of $P$ and $Q$ is a multi left ideal of $S \times S$.

Proof. Let $\left(x_{1}, x_{2}\right),\left(y_{1}, y_{2}\right) \in S \times S$. Then

$$
\begin{aligned}
\left(C_{P} \times C_{Q}\right)\left(\left(x_{1}, x_{2}\right)+\left(y_{1}, y_{2}\right)\right) & =\left(C_{P} \times C_{Q}\right)\left(x_{1}+y_{1}, x_{2}+y_{2}\right) \\
& =\min \left\{C_{P}\left(x_{1}+y_{1}\right), C_{Q}\left(x_{2}+y_{2}\right)\right\} \\
& \geq \min \left\{\min \left\{C_{P}\left(x_{1}\right), C_{P}\left(y_{1}\right)\right\}, \min \left\{C_{Q}\left(x_{2}\right), C_{Q}\left(y_{2}\right)\right\}\right\} \\
& =\min \left\{\min \left\{C_{P}\left(x_{1}\right), C_{Q}\left(x_{2}\right)\right\}, \min \left\{C_{P}\left(y_{1}\right), C_{Q}\left(y_{2}\right)\right\}\right\} \\
& =\min \left\{\left(C_{P} \times C_{Q}\right)\left(x_{1}, x_{2}\right),\left(C_{P} \times C_{Q}\right)\left(y_{1}, y_{2}\right)\right\}
\end{aligned}
$$

and

$$
\begin{aligned}
\left(C_{P} \times C_{Q}\right)\left(\left(x_{1}, x_{2}\right)\left(y_{1}, y_{2}\right)\right) & =\left(C_{P} \times C_{Q}\right)\left(x_{1} y_{1}, x_{2} y_{2}\right) \\
& =\min \left\{C_{P}\left(x_{1} y_{1}\right), C_{Q}\left(x_{2} y_{2}\right)\right\} \\
& \geq \min \left\{C_{P}\left(y_{1}\right), C_{Q}\left(y_{2}\right)\right\} \\
& =\left(C_{P} \times C_{Q}\right)\left(y_{1}, y_{2}\right) .
\end{aligned}
$$

Hence cartesian product of $P$ and $Q$ is a multi left ideal of $S \times S$.

Definition 3.12. A multi subset $P$ over a semiring $S$ is called multi bi-ideal if for all $x, y, z \in S$ we have

(i) $C_{P}(x+y) \geq \min \left\{C_{P}(x), C_{P}(y)\right\}$

(ii) $C_{P}(x y z) \geq \min \left\{C_{P}(x), C_{P}(z)\right\}$

Definition 3.13. Let $S$ be a semiring and $M$ be a multiset over $S$. Then the characteristic of the multiset $M$ is defined as $\chi_{M}(x)=\left\{\begin{array}{c}\infty \text { if } x \in M \\ 0 \text { if } x \notin M\end{array}\right.$

Definition 3.14. A multi subset $M$ over a semiring $S$ is called multi quasi-ideal if for all $x, y \in S$ we have

(i) $C_{M}(x+y) \geq \min \left\{C_{M}(x), C_{M}(y)\right\}$

(ii) $\left(C_{M} \circ \chi_{M}\right) \cap\left(\chi_{M} o C_{M}\right) \subseteq C_{M}$

Lemma 3.15. A multi subset $M$ over a semiring $S$ is a multi left (resp. right) ideal of $S$ if and only if for all $x, y \in S$, we have

(i) $C_{M}(x+y) \geq \min \left\{C_{M}(x), C_{M}(y)\right\}$

(ii) $\chi_{M} o C_{M} \subseteq C_{M}\left(\right.$ resp. $\left.C_{M} o \chi_{M} \subseteq C_{M}\right)$.

Proof. Assume that $M$ is a multi left ideal of $S$. Then it is sufficient to show that the condition (ii) is satisfied. Let $x \in S$. If $\left(\chi_{M} o C_{M}\right)(x)=0$, it is clear that $\left(\chi_{M} o C_{M}\right)(x) \leq C_{M}(x)$. Otherwise, there exist elements $a_{i}, b_{i} \in S$ 
and for $\mathrm{i}=1, \ldots, \mathrm{n}$ such that $x=\sum_{i=1}^{n} a_{i} b_{i}$. Then we have

$$
\begin{aligned}
\left(\chi_{M} \circ C_{M}\right)(x)= & \sup \left[\min _{i}\left\{\min \left\{\chi_{M}\left(a_{i}\right), C_{M}\left(b_{i}\right)\right\}\right\}\right] \\
& x=\sum_{i=1}^{n} a_{i} b_{i} \\
= & \sup \left[\min _{i}\left\{C_{M}\left(b_{i}\right)\right\}\right] \\
& x=\sum_{i=1}^{n} a_{i} b_{i} \\
\leq & \sup \left[\min _{i}\left\{C_{M}\left(a_{i} b_{i}\right)\right\}\right]=C_{M}(x) . \\
& x=\sum_{i=1}^{n} a_{i} b_{i}
\end{aligned}
$$

This implies that $\chi_{M} o C_{M} \subseteq C_{M}$.

Conversely, assume that the given conditions hold. Then it is sufficient to show the second condition of the definition of ideal. Let $x, y \in S$. Then we have

$$
\begin{aligned}
C_{M}(x y) \geq\left(\chi_{M} o C_{M}\right)(x y)= & \sup _{i}\left[\min _{i}\left\{\min \left\{\chi_{M}\left(a_{i}\right), C_{M}\left(b_{i}\right)\right\}\right\}\right] \geq C_{M}(y) . \text { Hence } C_{M} \text { is a multi left ideal of } S . \\
& x y=\sum_{i=1}^{n} a_{i} b_{i}
\end{aligned}
$$

The case for multi right ideal can be proved similarly.

Lemma 3.16. Any multi quasi-ideal of $S$ is a multi bi-ideal of $S$.

Proof. Let $M$ be any multi quasi-ideal of $S$. It is sufficient to show that $C_{M}(x y z) \geq \min \left\{C_{M}(x), C_{M}(z)\right\}$ and $C_{M}(x y) \geq \min \left\{C_{M}(x), C_{M}(y)\right\}$ for all $x, y, z \in S$.

In fact, by the assumption, we have

$$
\begin{aligned}
C_{M}(x y z) & \geq\left(\left(C_{M} o \chi_{M}\right) \cap\left(\chi_{M} o C_{M}\right)\right)(x y z) \\
= & \min \left\{\left(C_{M} o \chi_{M}\right)(x y z),\left(\chi_{M} o C_{M}\right)(x y z)\right\} \\
= & \min \left\{\quad \sup \left(\min \quad\left(C_{M}\left(a_{i}\right), \chi_{M}\left(b_{i}\right)\right)\right), \quad \sup \left(\min \quad\left(\chi_{M}\left(a_{i}\right), C_{M}\left(b_{i}\right)\right)\right)\right\} \\
& x y z=\sum_{i=1}^{n} a_{i} b_{i} a_{i} b_{i} \\
& \geq \min \left\{C_{M}(x), C_{M}(z)\right\}
\end{aligned}
$$

Similarly, we can show that $C_{M}(x y) \geq \min \left\{C_{M}(x), C_{M}(y)\right\}$.

\section{Multi Ideal of Regular and Intra-Regular Semiring}

Definition 4.1. A semiring $S$ is said to be regular if for each $x \in S$, there exists $a \in S$ such that $x=x a x$.

Lemma 4.2. A semiring $S$ is regular if and only if for any right ideal $R$ and any left ideal $L$ of $S$ we have $R L=R \cap L$.

Theorem 4.3. If semiring $S$ is regular then for any multi right ideal $M$ and any multi left ideal $N$ of $S$ we have $C_{M} \circ C_{N}=C_{M} \cap C_{N}$. 
Proof. Let $S$ be a regular semiring. By Lemma 3.5, we have $C_{M} \circ C_{N} \subseteq C_{M} \cap C_{N}$.

For any $x \in S$, there exist $a \in S$ such that $x=x a x$.

Then

$$
\begin{aligned}
\left(C_{M} o C_{N}\right)(x)= & \sup \left\{\min \left\{C_{M}\left(a_{i}\right), C_{N}\left(b_{i}\right)\right\}\right\} \\
& x=\sum_{i=1}^{n} a_{i} b_{i} \\
\geq & \min \left\{C_{M}(x a), C_{N}(x)\right\} \\
\geq & \min \left\{C_{M}(x), C_{N}(x)\right\} \\
& =\left(C_{M} \cap C_{N}\right)(x) .
\end{aligned}
$$

Therefore $\left(C_{M} \cap C_{N}\right) \subseteq\left(C_{M} o C_{N}\right)$.

Hence $\left(C_{M} o C_{N}\right)=\left(C_{M} \cap C_{N}\right)$.

Theorem 4.4. Let $S$ be a regular semiring. Then

(i) $C_{M} \subseteq C_{M} o \chi_{M} o C_{M}$ for every multi bi-ideal $M$ of $S$.

(ii) $C_{M} \subseteq C_{M}$ o $\chi_{M} o C_{M}$ for every multi quasi-ideal $M$ of $S$.

Proof. (i) Let $M$ be any multi bi-ideal of $S$ and $x$ be any element of $S$. Since $S$ is regular there exists $a \in S$ such that $x=x a x$.

$$
\begin{aligned}
\left(C_{M} o \chi_{M} o C_{M}\right)(x)= & \left.\sup \left(\min \left\{\left(C_{M} o \chi_{M}\right)\left(a_{i}\right), C_{M}\left(b_{i}\right)\right)\right\}\right) \\
& x=\sum_{i=1}^{n} a_{i} b_{i} \\
\geq & \min \left\{\left(C_{M} o \chi_{M}\right)(x a), C_{M}(x)\right\} \\
= & \min \left\{\min \left\{C_{M}(x), \chi_{M}(a)\right\}, C_{M}(x)\right\}=C_{M}(x)
\end{aligned}
$$

This implies that $C_{M} \subseteq C_{M} \circ \chi_{M} C_{M}$.

(i) $\Rightarrow$ (ii) This is straight forward from Lemma 3.16

Theorem 4.5. Let $S$ is a regular semiring. Then

(i) $C_{M} \cap C_{N} \subseteq C_{M} o C_{N} o C_{M}$ for every multi bi-ideal $M$ and every multi ideal $N$ of $S$.

(ii) $C_{M} \cap C_{N} \subseteq C_{M} o C_{N} o C_{M}$ for every multi quasi-ideal $M$ and every multi ideal $N$ of $S$.

Proof. (i) Let $M$ and $N$ be any multi bi-ideal and multi ideal of $S$, respectively and x be any element of $S$. Since $S$ is regular, there exists $a \in S$ such that $x=x a x$.

$$
\begin{aligned}
\left(C_{M} o C_{N} o C_{M}\right)(x)= & \sup \left\{\min \left\{\left(C_{M} o C_{N}\right)\left(a_{i}\right), C_{M}\left(b_{i}\right)\right\}\right\} \\
& x=\sum_{i=1}^{n} a_{i} b_{i} \\
\geq & \min \left\{\left(C_{M} o C_{N}\right)(x a), C_{M}(x)\right\} \\
= & \min \left\{\sup \left\{\min \left\{C_{M}\left(a_{i}\right), C_{N}\left(b_{i}\right)\right\}\right\}, C_{M}(x)\right\} \\
& \quad x a=\sum_{i=1}^{n} a_{i} b_{i} \\
\geq & \min \left\{\min \left\{C_{M}(x), C_{N}(a x a), C_{M}(x)\right\}(\text { since } x a=x a x a)\right. \\
\geq & \min \left\{C_{M}(x), C_{N}(x)\right\}=\left(C_{M} \cap C_{N}\right)(x)
\end{aligned}
$$


(i) $\Rightarrow$ (ii) This is straight forward from Lemma 3.16.

Definition 4.6. A semiring $S$ is said to be intra-regular if for each $x \in S$, there exist $a, b \in S$, such that $x=a x x b$.

Theorem 4.7. Let $S$ be a intra-regular semiring. Then $C_{M} \cap C_{N} \subseteq C_{M} o C_{N}$ for every multi left ideal $C_{M}$ and every multi right ideal $C_{N}$ of $S$.

Proof. Suppose $S$ is regular semiring. Let $M$ and $N$ be any multi left ideal and multi right ideal of $S$ respectively. Now let $x \in S$. Then by hypothesis there exist $a, b \in S$, such that $x=a x x b$. Therefore

$$
\begin{aligned}
\left(C_{M} o C_{N}\right)(x)= & \sup \left[\min _{i}\left\{\min \left\{C_{M}\left(a_{i}\right), C_{N}\left(b_{i}\right)\right\}\right\}\right] \\
& x=\sum_{i=1}^{n} a_{i} b_{i} \\
\geq & \min _{i}\left[\min \left\{C_{M}(a x), C_{N}(x b)\right\}\right] \\
\geq & \min \left\{C_{M}(x), C_{N}(x)\right\}=\left(C_{M} \cap C_{N}\right)(x)
\end{aligned}
$$

Theorem 4.8. Let $S$ be both regular and intra-regular semiring. Then

(ii) $C_{M}=C_{M} o C_{M}$ for every multi bi-ideal $M$ of $S$.

(ii) $C_{M}=C_{M} o C_{M}$ for every multi quasi-ideal $M$ of $S$.

Proof. (i) Let $x \in S$ and $M$ be any multi bi-ideal of $S$. Since $S$ is both regular and intra-regular there exist $a, b \in S$, such that $x=x a x x b x$. Therefore

$$
\begin{aligned}
\left(C_{M} o C_{M}\right)(x)= & \sup \left[\min _{i}\left\{\min \left\{C_{M}\left(a_{i}\right), C_{M}\left(b_{i}\right)\right\}\right\}\right] \\
& x=\sum_{i=1}^{n} a_{i} b_{i} \\
\geq & \min _{x=x a x x b x}\left\{C_{M}(x a x), C_{M}(x b x)\right\} \\
\geq & C_{M}(x) .
\end{aligned}
$$

Now $C_{M} o C_{M} \subseteq C_{M} \cap C_{M}=C_{M}$. Hence $C_{M} o C_{M}=C_{M}$ for every multi bi-ideal $M$ of $S$.

(i) $\Rightarrow$ (ii) This is straightforward from the Lemma 3.16.

Theorem 4.9. Let $S$ be a regular and intra-regular semiring. Then

(i) $C_{M} \cap C_{N} \subseteq C_{M} o C_{N}$ for all multi bi-ideals $M$ and $N$ of $S$.

(ii) $C_{M} \cap C_{N} \subseteq C_{M} o C_{N}$ for every multi bi-ideals $M$ and every multi quasi-ideal $N$ of $S$.

(iii) $C_{M} \cap C_{N} \subseteq C_{M} o C_{N}$ for every multi quasi-ideals $M$ and every multi bi-ideal $N$ of $S$.

(iv) $C_{M} \cap C_{N} \subseteq C_{M} o C_{N}$ for all multi quasi-ideals $M$ and $N$ of $S$. 
Proof. (i) Let $x \in S$ and $M$ be any multi bi-ideal of $S$. Since $S$ is both regular and intra-regular there exist $a, b \in S$, such that $x=x a x x b x$. Therefore

$$
\begin{aligned}
\left(C_{M} C_{N}\right)(x)= & \sup \left[\min _{i}\left\{\min \left\{C_{M}\left(a_{i}\right), C_{N}\left(b_{i}\right)\right\}\right\}\right] \\
& x=\sum_{i=1}^{n} a_{i} b_{i} \\
\geq & \min \left\{C_{M}(x a x), C_{N}(x b x)\right\} \\
\geq & \min \left\{C_{M}(x), C_{N}(x)\right\}=\left(C_{M} \cap C_{N}\right)(x)
\end{aligned}
$$

which implies $C_{M} \cap C_{N} \subseteq C_{M} o C_{N}$.

$(i) \Rightarrow(i i) \Rightarrow(i v)$ and $(i) \Rightarrow(i i i) \Rightarrow(i v)$ are obvious from Lemma 3.16.

\section{REFERENCES}

[1] W. D. Blizard, Multiset theory, Notre Dame J. Form. Log. 30 (1989), 36-66.

[2] K. P. Girish and S.J. John, Relations and Functions in Multiset Context, Inform. Sci. 179 (6) (2009), 758-768.

[3] K. P. Girish and S. J. John, General Relations between partially ordered multisets and their chains and antichains, Math. Commun. 14(2) (2009), 193-206.

[4] J.S.Golan, Semirings and their applications, Kluwer Academic Publishers,1999.

[5] A. Hallez, A. Bronselaer, G. D. Tre, Comparison of sets and multisets, Int. J. Uncertain. Fuzzy. Knowl. Based Syst. 17 (Suppl. 1) (2009), 153-172.

[6] J. L. Hickman, A note on the concept of multiset, Bull. Aust. Math. Soc. 22 (1980), 211-217.

[7] S. Miyamoto, Operations for real-valued bags and bag relations, in ISEA-EUSFLAT, (2009), 612-617.

[8] S. K. Nazmul, P. Majumdar and S. K. Samanta, On multisets and multigroups, Ann. Fuzzy Math. Inform. 6(3) (2013), $643-656$.

[9] D. Singh, A. M. Ibrahim, T. Yohanna and J. N. Singh, An overview of the applications of multisets, Novi Sad J. Math. 37 (2) (2007), $73-92$.

[10] R. R. Yager, On the theory of bags, Int. J. Gen. Syst. 13(1986), 23-37. 